\title{
Efemp1 modulates elastic fiber formation and mechanics of the extrahepatic bile duct
}

Jessica Llewellyn ${ }^{1,2}$, Emilia Roberts ${ }^{3}$, Chengyang Liu ${ }^{4}$, Ali Naji ${ }^{4}$, Richard K. Assoian ${ }^{2,3,5}$, Rebecca G. Wells ${ }^{1,2^{*}}$

1. Department of Medicine, The University of Pennsylvania, Philadelphia, PA, 19104, USA. 2. NSF Center

for Engineering MechanoBiology, The University of Pennsylvania, Philadelphia, PA, 19104, USA. 3. Program in Translational Biomechanics, Institute of Translational Medicine and Therapeutics, The University of Pennsylvania, Philadelphia, PA, 19104, USA. 4. Department of Surgery, The University of Pennsylvania, Philadelphia, PA, 19104, USA. 5. Department of Systems Pharmacology and Translational Therapeutics, The University of Pennsylvania, Philadelphia, PA, 19104, USA.

*rgwells@pennmedicine.upenn.edu

\section{Abstract}

EGF-Containing Fibulin Extracellular Matrix Protein 1 (EFEMP1, also called fibulin 3) is an extracellular matrix protein linked in a genome-wide association study to biliary atresia, a fibro-inflammatory disease of the neonatal extrahepatic bile duct. EFEMP1 is expressed in most tissues and Efemp1 null mice have decreased elastic fibers in visceral fascia; however, in contrast to other short fibulins (fibulins 4 and 5), EFEMP1 does not have a role in the development of large elastic fibers, and its overall function remains unclear. We demonstrated that EFEMP1 is expressed in the submucosa of both neonatal and adult mouse and human extrahepatic bile ducts and that, in adult $E f e m p 1^{+/}$mice, elastin organization into fibers is decreased. We used pressure myography, a technique developed to study the mechanics of the vasculature, to show that $E f e m p 1^{+/-}$extrahepatic bile ducts are more compliant to luminal pressure, leading to increased circumferential stretch. We conclude that EFEMP1 has an important role in the formation of elastic fibers and mechanical properties of the extrahepatic bile duct. These data suggest 
that altered expression of EFEMP1 in the extrahepatic bile duct leads to an abnormal response to mechanical stress such as obstruction, potentially explaining the role of EFEMP1 in biliary atresia.

Key words: fibulin 3; bile duct mechanics; biliary atresia; pressure myography; collagen; elastin

Abbreviations: EFEMP1, EGF-Containing Fibulin Extracellular Matrix Protein 1; SHG, second harmonic generation; DAPI, 4',6-diamidino-2-phenylindole; WT, wild type; PBS, phosphate buffered saline; SEM, standard error of the mean

\section{Introduction}

Biliary atresia is a rare and severe pediatric disease that initially causes fibrosis, obstruction, and obliteration of the extrahepatic bile ducts but rapidly leads to liver fibrosis and end-stage liver disease. The pathogenesis of biliary atresia is poorly understood. The inciting event appears to be exposure to an environmental agent such as a toxin ${ }^{[1,2]}$ or virus ${ }^{[3,4]}$ during gestation in a fetus with developmental and genetic susceptibility ${ }^{[5-7]}$. Although no single gene has been found to be causative, multiple genes have been associated with biliary atresia in large population studies ${ }^{[8-16]}$. One such gene is EFEMP1, which was identified in a genome-wide association study in two independent European-American biliary atresia patient cohorts as a candidate susceptibility gene ${ }^{[12]}$.

EGF-Containing Fibulin Extracellular Matrix Protein 1 (EFEMP1), also known as fibulin-3, is member of the fibulin family of proteins. These proteins interact with a range of different matrix proteins, contributing to ECM organization and stability ${ }^{[17]}$. Fibulin-3 is part of the subgroup of short fibulins that includes fibulins $-4,-5$ and -7 , although its function is less well described. Fibulins- 4 and -5 have critical roles in the formation of elastic fibers, particularly in large vessels. An elastic fiber is composed of an elastin core embedded in a fibrillin-rich microfibril scaffold. Its formation is a complex multistage process, with more 
than 30 elastic-fiber associated proteins identified ${ }^{[18]}$. Fibulin-4 deletions in mice cause perinatal lethality due to aortic rupture ${ }^{[19,20]}$. Fibulin-5 deletion leads to irregular aggregation of elastin and lack of the normal characteristic lamellar structures in mouse aortas ${ }^{[21,22]}$. In contrast, fibulin-3 has a relatively low binding affinity for tropoelastin, the elastin monomer and, although fibulin 3 is expressed in lung and aorta, the large elastic fibers in these organs appear normal in Efemp1 null mice ${ }^{[17,23]}$. Efemp1 null mice instead demonstrate a loss of the fine elastic fibers found in fascia, with fascial herniation, early aging and low reproductive capacity, suggestive of a tissue- or elastic fiber size-specific function of EFEMP1. Here we establish a role for EFEMP1 in the formation of elastic fibers in the extrahepatic bile duct and demonstrate the mechanical importance of EFEMP1 in this tissue.

\section{Results}

\section{EFEMP1 is highly expressed during the postnatal development of the extrahepatic bile duct in mouse}

\section{and human}

The expression pattern of EFEMP1 during the postnatal development of the mouse and human extrahepatic bile duct was assessed by immunostaining (Fig. 1A). In the neonatal mouse (P2) and human (3-day-old) extrahepatic bile duct, EFEMP1 is found highly expressed in both biliary epithelial cells and fibroblasts. In adult extrahepatic bile ducts however, EFEMP1 is mainly found deposited in the extrahepatic bile duct interstitium, with limited expression in cells. To evaluate a potential role for EFEMP1 in elastic fiber formation, we stained for both elastin and elastic fibers. Elastin deposition occurred later during postnatal development than EFEMP1 and was first apparent starting at P5, while elastic fibers only became visible between P10 and P14 (Fig. 1B). Interestingly although EFEMP1, elastin and elastic fibers are found throughout the extrahepatic bile duct submucosa, a strong deposition under the biliary epithelial layer is apparent in adult extrahepatic bile ducts (Fig. 1A-B). The sequential presence and similar distribution suggest a potential interaction between EFEMP1 and elastin. 


\section{Efemp ${ }^{+/-}$extrahepatic bile ducts have reduced elastic fibers}

To evaluate whether EFEMP1 has a role in elastic fiber formation in the extrahepatic bile duct, we compared expression of elastin and organized elastic fibers in the extrahepatic bile ducts of Efemp $1^{+/}$and WT mice. (Although Efemp1 null mice were originally reported to be viable ${ }^{[23]}$, Efemp $^{-/-}$mice were not born in our colony.) The amount of elastin deposition in the submucosa of the extrahepatic bile duct is comparable in the WT and $\mathrm{Efemp1}^{+/}$mice, in both covering approximately $5 \%$ of the extrahepatic bile duct area; however, the distribution is different, with decreased localization under the biliary epithelial cell monolayer in the Efemp1 ${ }^{+-}$(Fig. 2A). The number of elastic fibers present in the extrahepatic bile duct submucosa is reduced by nearly half from 12.7 fibers $/ 50 \mu \mathrm{m}^{2}$ in the WT to 6.6 fibers $/ 50 \mu \mathrm{m}^{2}$ in the Efemp $^{+/-}$mice (Fig. 2B). This suggests that EFEMP1 has no impact on the expression and deposition of elastin but plays a role in the formation of elastic fibers. The expression of collagen, the other key structural protein in the extrahepatic bile duct, was examined using second harmonic generation (SHG) imaging, but both the amount of collagen and wavelength of collagen fiber crimps were similar in WT and Efemp $1^{+/-}$extrahepatic bile ducts (Fig. 2C, Supplemental Fig. 1).

\section{Efemp $1^{+/-}$extrahepatic bile ducts are more compliant to luminal pressure}

To determine the functional relevance of the changes in the extrahepatic bile duct submucosa observed with depletion of EFEMP1, we measured the mechanical properties of the extrahepatic bile duct using pressure myography. This technique, which was developed for and is typically used to study vessels, measures the changes in the diameter of a cylindrical structure in response to luminal pressure ${ }^{[24-27]}$. When we compared the response of adult $E f e m p 1^{+/-}$and WT extrahepatic bile ducts, we found that the Efemp $^{+/-}$ducts expanded more in response to pressure, leading to an increased outer diameter at any given pressure including at normal physiological pressures $(0.8-3.5 \mathrm{mmHg}$ ) and at pressures found in obstructed ducts (12-15 mmHg) (Fig. 3A), using pressure values taken from the literature ${ }^{[28-31]}$. Analysis 
was done on physiologically relevant pressures although data to $60 \mathrm{mmHg}$ were collected (Fig. S2). The derived circumferential stretch-stress curve revealed a significant increase in stretch of the duct, while no statistically significant difference in stress was observed between WT and Efemp1 ${ }^{+/}$extrahepatic bile ducts (Fig. 3B). The stress-stretch curve has a similar shape as that of the aorta, in which the first phase is associated with elastin and the second with collagen ${ }^{[32,33]}$. The difference in stretch between WT and Efemp1 $1^{+-}$extrahepatic bile ducts occurs in the first low pressure phase as demonstrated by the $24.6 \%$ difference in luminal diameter between WT extrahepatic bile duct $(75.5 \pm 4.118 \mu \mathrm{m})$ and $E f e m p 1^{+/}$ extrahepatic bile ducts $(94.09 \pm 8.239 \mu \mathrm{m})$ in the loaded (pressurized) state (Fig. 3C). The system is pressurized (unloaded to loaded) to near $0 \mathrm{mmHg}(0.1 \mathrm{mmHg})$ at the start of each pressure myography test. The tangential modulus of the stress-stretch curve, an estimate of tissue stiffness, was also calculated, and the results indicated that stiffness of the extrahepatic bile duct significantly increases with increasing filling pressures. At physiologically relevant pressures, normal pressure $(2 \mathrm{mmHg})$ and obstruction pressure $(12 \mathrm{mmHg})$, the stiffnesses of WT and Efemp $1^{+/-}$extrahepatic bile ducts are similar (Fig. 3D). These experiments demonstrate that EFEMP1 has a functional role in extrahepatic bile duct mechanics, in particular the circumferential stretch response to physiological filling pressures.

\section{Discussion}

We have shown here that EFEMP1 is important for the formation of elastic fibers in the extrahepatic bile duct and contributes to duct mechanical properties, playing a significant role in the response to luminal pressure. These findings provide a potential mechanistic explanation for the link between EFEMP1 and biliary atresia identified in a genome-wide association study ${ }^{[12]}$, given that a controlled response to intraductal pressures and ability to resist obstruction are likely important in the function of the normal and injured duct. 
Although it is likely to have a wide range of functions, EFEMP1 has been implicated in the formation and turnover of elastic fibers in a highly tissue-specific manner ${ }^{[34]}$. The sequential expression of EFEMP1, elastin and then elastic fibers in postnatal development, the co-localization of the three under the biliary epithelial layer, and the reduction of the number of fibers in Efemp1 $1^{+-}$mice all suggest that EFEMP1 is important for the formation of elastic fibers in the extrahepatic bile duct. The lack of EFEMP1 expression in cells of the adult extrahepatic bile duct suggests that its role in elastic fiber homeostasis may be limited in the uninjured adult, although the mechanism by which EFEMP1 regulates elastic fibers requires additional investigation. EFEMP1 binds weakly to tropoelastin but does not bind fibrillin 1, the major component of the microfibril surrounding elastin ${ }^{[17,35]}$, and in the Efemp $1^{\%}$ mouse, electron microscopy of the skin showed that the dark elastin core of elastic fibers was fragmented ${ }^{[23]}$. This points towards a role for EFEMP1 in the formation of the elastin core of the elastic fiber.

To our knowledge, this study is the first to use pressure myography to test the mechanics of a non-vascular tissue. The extrahepatic biliary system is under very low pressure in vivo, particularly when compared to arteries, as reflected in the lack of thick elastin lamellae or a smooth muscle layer. To adapt the technique to the extrahepatic bile duct, lower pressures of 0-60 $\mathrm{mmHg}$ were used. Interestingly, while structure and pressures differ, the stretch experienced by the extrahepatic bile ducts is within the same range as experienced by arteries (stretch less than 2 fold) and both show a similar bi-phasic stress-strain curve. The initial phase has been attributed to the contribution of elastin and the second to collagen ${ }^{[32,33]}$. Given the similar degrees of stretch experienced by the extrahepatic bile duct and arteries, the two phases are likely to be similar for both structures. The curves for WT versus $E f e m p 1^{+/-}$ducts show a difference in stretch which we attribute to changes in elastin; however, the second phases are remarkably similar, reflecting the similar collagen distribution and organization in the two genotypes.

Appropriate responses to mechanical stresses are key to maintaining tissue integrity and normal cell function. EFEMP1 has recently been linked to biliary atresia in a genome-wide association study. Our data 
show that even at physiological pressures the extrahepatic bile duct undergoes significant stretch, which we predict would affect both biliary epithelial cells and interstitial fibroblasts. Neonatal biliary epithelial cells in particularly have relatively immature tight junctions ${ }^{[36]}$, and the increased stretch they experience in the setting of EFEMP1 abnormalities could result in the leakage of toxic bile into the submucosa, causing injury. Increased stretch could also impact submucosal fibroblasts, as fibroblasts in general are highly mechanosensitive cells and in tissues like skin and tendon show responses to stretch that include increased collagen 1 expression ${ }^{[37]}$. Alternatively, the increased stretch observed with reduced EFEMP1 could serve a protective function, preventing the upstream (hepatic) consequences of obstruction.

In summary, EFEMP1 is involved in the formation of elastic fibers, which provide tensile strength limiting excessive mechanical disruptions to the tissue. An inability to respond appropriately to both physiological and obstruction pressures is likely to lead to cellular dysfunction and reduced ECM integrity. Although the predicted high binding promiscuity of EFEMP1 ${ }^{[34]}$ suggests that it has as-yet-undescribed functions, the structural role of EFEMP1 in the extrahepatic bile duct may explain its link to biliary atresia.

\section{Experimental procedures}

\section{Human tissue}

Anonymized fresh human extrahepatic bile duct was obtained as part of the Human Pancreas

Procurement and Analysis Program (HPPAP), which was granted IRB exemption (protocol 826489).

Samples were taken after the unexpected deaths of otherwise healthy individuals and were formalinfixed and embedded after receipt.

\section{Mouse tissue}

All work with mice was in accordance with protocols approved by the University of Pennsylvania Institutional Animal Care and Use Committee, as per the National Institutes of Health Guide for the Use 
and Care of Animals. C57BI/6j mice were obtained from the Jackson Laboratory (Bar Harbor, ME, USA). Efemp $1^{+/-}$mice were a gift from Lihua Marmorstein (University of Arizona) ${ }^{[23]}$. Efemp $1^{+/-}$pairs were bred, however, although the nulls are reported to be viable, only $E f e m p 1^{+/-}$and $E f e m p 1^{+/+}$offspring were born in our colony and the heterozygotes were used for all experiments. Extrahepatic bile ducts were dissected from mice at different ages as noted in the figures and were formalin fixed (10\%), paraffin embedded and sectioned at $5 \mu \mathrm{m}$ thickness. Both male and female mice were used for all analyses.

\section{Immunofluorescence, elastic fiber staining and second harmonic generation imaging}

For immunofluorescence of mouse and human extrahepatic bile ducts, samples were dewaxed with xylene and rehydrated through a graded series of alcohols and distilled water. Heat-mediated antigen retrieval with $10 \mathrm{mM}$ citric acid pH 6.0 in a pressure cooker for 2 hrs was used for staining with antibodies against EFEMP1 (1:100, Thermo Fisher, PA5-29347, Waltham, MA); 0.5\% hyaluronidase (Sigma, H3506 type I-S, St-Louis, MO) treatment for $60 \mathrm{~min}$ at $37^{\circ} \mathrm{C}$ was used for staining with antibodies against elastin (1:100, Cedarlane, CL55041AP, Burlington, NC). Sections were blocked with StartingBlock ${ }^{\mathrm{TM}}$ T20/phosphate buffered saline (PBS) Blocking Buffer (Thermo Scientific, Waltham, MA) before being incubated with primary antibodies (in $0.2 \%$ Triton $\mathrm{X}-100,3 \%$ serum, in PBS) at $4^{\circ} \mathrm{C}$ overnight. Sections were then incubated with Cy3-conjugated anti-rabbit secondary antibody for 60 min (1:500, Vector Laboratories, Burlington, CA), DAPI for 10 min (4', 6-Diamidino-2-Phenylindole, Dihydrochloride) and were mounted. Stained sections were imaged using a Zeiss Axio Observer 7 inverted microscope and ZEN blue software. For elastic fiber staining, paraffin sections were dewaxed and rehydrated and stained using an Elastic Stain Kit (Millipore, HT25A, Burlington, MA). Brightfield imaging was done on a Nikon E600 microscope with Nikon NIS-Elements software. For SHG imaging, paraffin sections were imaged using a Leica SP8 confocal/multiphoton microscope and Coherent Chameleon Vision II Ti:Sapphire laser (Leica, Buffalo Grove, IL) tuned to a wavelength of $910 \mathrm{~nm}$. 


\section{Image analysis}

Raw image files from immunofluorescence and SHG imaging were processed using Fiji ImageJ software ${ }^{[38]}$. Quantification of the percent area stained was assessed using the threshold and percent area functions. The number of elastic fibers per $50 \mu \mathrm{m}^{2}$ was counted manually using the Count tool. The crimp wavelength and depth were measured manually using the Line tool. For all analyses, 3-5 images per sample were selected. Analyses were done blinded.

\section{Pressure myography}

Extrahepatic bile ducts were isolated from adult (10-15 week old) WT and Efemp1 ${ }^{+/-}$mice and placed in Hank's balanced salt solution (HBSS) without calcium and magnesium at $37^{\circ} \mathrm{C}$. The extrahepatic bile ducts were then carefully placed onto two $385 \mu \mathrm{m}$ stainless steel cannulas on a DMT model 114P Pressure Myograph (DMT-USA, Ann Arbor, MI) and tied into place with 6-0 silk sutures (SUT-S 104, Braintree Scientific Inc., Braintree, MA), creating a closed system. The mounted ducts were stretched until straight, the force transducer was brought to zero, and then the ducts were stretched axially to $1 \mathrm{mN}$ of force to standardize baseline axial conditions. The system was pressurized using medical air (AI USP 300; Airgas USA, Cherry Hill, NJ) and the extrahepatic bile ducts were preconditioned for $15 \mathrm{~min}$ at $40 \mathrm{mmHg}$ before being pressurized in a stepwise manner from $0-20 \mathrm{mmHg}$ in $2 \mathrm{mmHg}$ increments and then to $60 \mathrm{mmHg}$ in $5 \mathrm{mmHg}$ increments. The system was fitted with a Nikon Diaphot inverted microscope, enabling measurement of the vessel length (VL), wall thickness $(T)$ and outer diameter (OD) using MYOVIEW software (DMT-USA, Ann Arbor, MI). This was done 3 times on each extrahepatic bile duct and values were averaged for data analysis. No difference in initial diameter of pressurized extrahepatic bile ducts (0.1 $\mathrm{mmHg}$ ) was observed amongst tests for a given duct, suggesting that duct returns to normal after each pressurization. Axial stretch measurements were not performed as these are unlikely to be forces experienced by the extrahepatic bile duct due to the low pressures of the system. Real-time imaging and 
the MYOVIEW software were used for direct measurement of the loaded outer diameters $\left(2 a_{o}\right)$. From this, the unloaded inner radius $\left(A_{i}\right)$ was calculated as $A_{i}=A_{0^{-}} \mathrm{H}$, where $\mathrm{H}$ is the unloaded wall thickness. The duct volume $(V)$ was used to determine the loaded inner radii using $a_{i}=\sqrt{a_{0}^{2}-(V / \pi l)}$ where $\mathrm{I}$ is the length between the inner sutures and measured using calipers. The loaded wall thickness $(h)$ calculated using $h=a_{0}-a_{i}$. The circumferential stretch $\left(\lambda_{\theta}\right)$ was calculated using $\lambda_{\theta}=\left(a_{i}+h / 2\right) /\left(A_{1}+H / 2\right)$ and circumferential stress using $\sigma_{\vartheta}=\left(P a_{i}\right) / h$ where $\mathrm{P}$ is pressure. As the extrahepatic bile duct shows a nonlinear stress-stretch relationship, the tangent moduli (slope) were calculated as a measure of extrahepatic bile duct stiffness. An exponential curve was fitted to the stress-stretch curve using MATLAB (R2021a, curve fitting tool). The tangent modulus was obtained by taking the derivative of the stress-stretch curves at normal physiological $(2 \mathrm{mmHg})$ and obstruction $(12 \mathrm{mmHg})$ pressures.

\section{Statistical analysis}

Statistical significance was calculated with Prism 9 (GraphPad software, LaJolla, CA). The image analysis data were assessed by unpaired T-test. Statistical significance of the pressure myography was assessed by two-way ANOVA and Šidák post hoc analysis. Significant $(p<0.05)$ results are noted on figures.

\section{Author Contributions:}

J.L. contributed to study concept and design, data acquisition, analysis and interpretation, and drafting of the manuscript. E.R. contributed to acquisition, analysis, and interpretation of data. A.N. and C.L provided material support and contributed to data interpretation. R.K.A contributed to study concept and design and participated in data interpretation. R.G.W. conceived ideas and designed the research, provided critical revision of the manuscript, obtained funding and supervised the study.

\section{Declaration of Competing Interests:}


None

\section{Funding and Acknowledgments:}

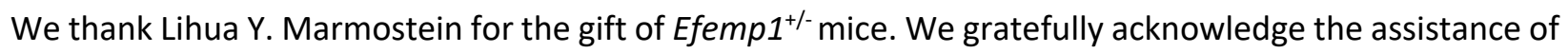
the following core facilities and individuals at the University of Pennsylvania: the Penn Vet Imaging Core (NIH grant S10 OD021633-01) and Gordon Ruthel, the Perelman School of Medicine Cell and Developmental Biology Microscopy Core, and the NIDDK Center for Molecular Studies in Digestive and Liver Diseases Molecular Pathology and Imaging Core (NIH P30 DK050306). E.R. was supported by the Program in Translational Biomechanics of the Institute for Translational Medicine and Therapeutics at the University of Pennsylvania. Funding was provided by NIH R01 DK119290 (to R.G.W.); NIH grant UC4 DK112217 (to A.N.) and the NIDDK/City of Hope Integrated Islet Distribution Program (IIDP) Inst. \#10039645; the Center for Engineering MechanoBiology (CEMB), an NSF Science and Technology Center, under grant agreement CMMI: 15-48571 (to R.G.W. and R.K.A.); and the Fred and Suzanne Biesecker Pediatric Liver Center (to R.G.W.).

\section{References}

1. Harper, P. Plant, J.W. Unger, D.B. Congenital biliary atresia and jaundice in lambs and calves. Aust Vet J. 67(1):18-22 (1990).

2. Lorent, K. et al. Identification of a plant isoflavonoid that causes biliary atresia. Sci Transl Med. 7(286):286ra67; 10.1126/scitranslmed.aaa1652. (2015).

3. Landing, B.H. Considerations of the pathogenesis of neonatal hepatitis, biliary atresia and choledochal cyst--the concept of infantile obstructive cholangiopathy. Prog Pediatr Surg. 6:113-39 (1974).

4. Averbukh, L.D. Wu, G.Y. Evidence for Viral Induction of Biliary Atresia: A Review. J Clin Transl Hepatol. 6(4):410-9 (2018).

5. Harpavat, S. Finegold, M.J. Karpen, S.J. Patients with biliary atresia have elevated direct/conjugated bilirubin levels shortly after birth. Pediatrics. 128(6):e1428-33; 10.1542/peds.2011-1869 (2011).

6. Bezerra, J.A. et al. Biliary Atresia: Clinical and Research Challenges for the Twenty-First Century. Hepatology. 68(3):1163-73 (2018).

7. Kilgore, A. Mack, C.L. Update on investigations pertaining to the pathogenesis of biliary atresia. Pediatr Surg Int. 33(12):1233-41 (2017).

8. Liu, F. et al. PDGFA gene rs9690350 polymorphism increases biliary atresia risk in Chinese children. Biosci Rep.40(7) BSR20200068; 10.1042/BSR20200068. (2020)

9. Garcia-Barceló, M.M. et al. Genome-wide association study identifies a susceptibility locus for biliary 
atresia on 10q24.2. Hum Mol Genet. 19(14):2917-25 (2010).

10. Leyva-Vega, M. et al. Genomic alterations in biliary atresia suggest region of potential disease susceptibility in 2q37.3. Am J Med Genet A. 152A(4):886-95 (2010).

11. Ningappa, M. et al. The Role of ARF6 in Biliary Atresia. PLoS One. 10(9):e0138381; 10.1371/journal.pone.0138381 (2015).

12. Chen, Y. et al. A genome-wide association study identifies a susceptibility locus for biliary atresia on 2p16.1 within the gene EFEMP1. PLoS Genet. 14(8):e1007532; 10.1371/journal.pgen.1007532 (2018).

13. Cui, S. et al. Evidence from human and zebrafish that GPC1 is a biliary atresia susceptibility gene. Gastroenterology. 144(5):1107-15.e3 (2013).

14. Kaewkiattiyot, S. Honsawek, S. Vejchapipat, P. Chongsrisawat, V. Poovorawan, Y. Association of Xprolyl aminopeptidase 1 rs17095355 polymorphism with biliary atresia in Thai children. Hepatol Res. 41(12):1249-52 (2011).

15. Rajagopalan, R. et al. Exome Sequencing in Individuals with Isolated Biliary Atresia. Sci Rep. 10(1):2709; 10.1038/s41598-020-59379-4 (2020).

16. Cheng, G. et al. Common genetic variants regulating ADD3 gene expression alter biliary atresia risk. J Hepatol. 59(6):1285-91 (2013).

17. Kobayashi, N. et al. A comparative analysis of the fibulin protein family. Biochemical characterization, binding interactions, and tissue localization. J Biol Chem. 282(16):11805-16 (2007).

18. Kielty, C.M. Sherratt, M.J. Shuttleworth, C.A. Elastic fibres. J Cell Sci. 115(Pt 14):2817-28 (2002).

19. Horiguchi, M. et al. Fibulin-4 conducts proper elastogenesis via interaction with cross-linking enzyme lysyl oxidase. Proc Natl Acad Sci U S A. 106(45):19029-34 (2009).

20. Huang, J. et al. Fibulin-4 deficiency results in ascending aortic aneurysms: a potential link between abnormal smooth muscle cell phenotype and aneurysm progression. Circ Res. 106(3):583-92 (2010).

21. Yanagisawa, H. et al. Fibulin-5 is an elastin-binding protein essential for elastic fibre development in vivo. Nature. 415(6868):168-71 (2002).

22. Nakamura, T. et al. Fibulin-5/DANCE is essential for elastogenesis in vivo. Nature. 415(6868):171-5 (2002).

23. McLaughlin, P.J. et al. Lack of fibulin-3 causes early aging and herniation, but not macular degeneration in mice. Hum Mol Genet. 16(24):3059-70 (2007).

24. Brankovic, S. Hawthorne, E.A. Yu, X. Zhang, Y. Assoian, R.K. MMP12 preferentially attenuates axial stiffening of aging arteries. J Biomech Eng. 141(8): 0810041-0810049 (2019).

25. Wenceslau, C.F. et al. Guidelines for the measurement of vascular function and structure in isolated arteries and veins. Am J Physiol Heart Circ Physiol. 321(1):H77-H111 (2021).

26. Mulvany, M.J. Halpern, W. Contractile properties of small arterial resistance vessels in spontaneously hypertensive and normotensive rats. Circ Res. 41(1):19-26 (1977).

27. Wagenseil, J.E. Nerurkar, N.L. Knutsen, R.H. Okamoto, R.J. Li, D.Y. Mecham, R.P. Effects of elastin haploinsufficiency on the mechanical behavior of mouse arteries. Am J Physiol Heart Circ Physiol. 289(3): H1209-17 (2005).

28. Wiener, S.M. et al. Manometric changes during retrograde biliary infusion in mice. Am J Physiol Gastrointest Liver Physiol. 279(1):G49-66 (2000).

29. Beltrán, M.A. Beltrán, A.A. Common bile duct pressure in patients with and without cholelithiasis: A case-control study. J Hepatobiliary Pancreat Sci. 28(5):443-9 (2021).

30. Yang, J. Lu, B. Establishment of a novel rat model of severe acute cholangitis. Iran J Basic Med Sci. 18(11):1124-9 (2015).

31. Heinrich, S. Georgiev, P. Weber, A. Vergopoulos, A. Graf, R. Clavien, P.A. Partial bile duct ligation in mice: a novel model of acute cholestasis. Surgery. 149(3):445-51 (2011).

32. Wagenseil, J.E. Mecham, R.P. Elastin in large artery stiffness and hypertension. J Cardiovasc Trans/ Res. 5(3):264-73 (2012). 
33. Carta, L. et al. Discrete contributions of elastic fiber components to arterial development and mechanical compliance. Arterioscler Thromb Vasc Biol. 29(12):2083-9 (2009).

34. Livingstone, I. Uversky, V.N. Furniss, D. Wiberg, A. The Pathophysiological Significance of Fibulin-3. Biomolecules. 10(9) 1294; 10.3390/biom10091294 (2020).

35. El-Hallous, E. et al. Fibrillin-1 interactions with fibulins depend on the first hybrid domain and provide an adaptor function to tropoelastin. J Biol Chem. 282(12):8935-46 (2007).

36. Khandekar, G. et al. Coordinated development of the mouse extrahepatic bile duct: implications for neonatal susceptibility to biliary injury J Hepatol. 72(1):135-145 (2020).

37. Wang, J.H. Thampatty, B.P. Lin, J.S. Im, H.J. Mechanoregulation of gene expression in fibroblasts. Gene. 391(1-2):1-15 (2007).

38. Schindelin, J. et al. Fiji: an open-source platform for biological-image analysis. Nat Methods. 9(7):676-82 (2012). 

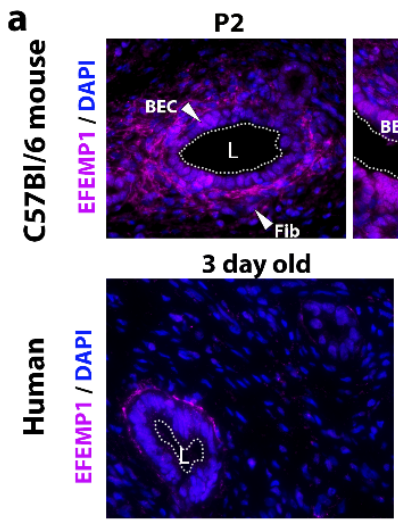

b

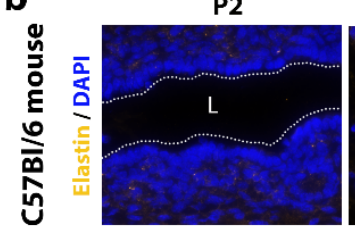

C

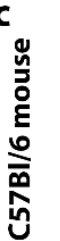

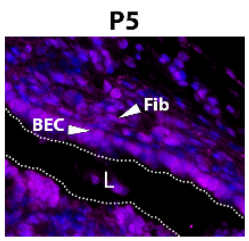

6 month old

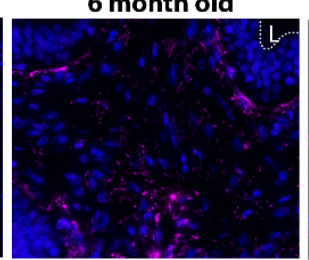

P5

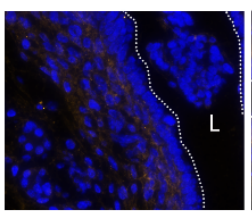

P5

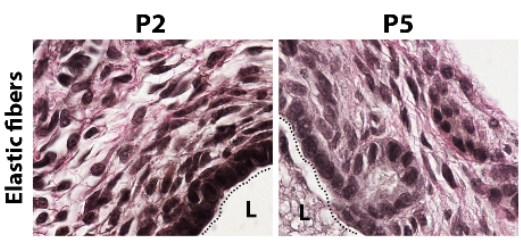

P7

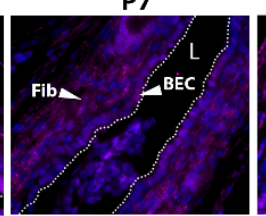

3 year old

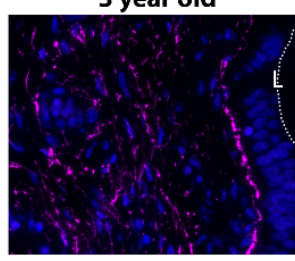

P7
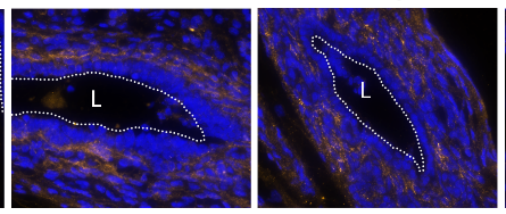

P10

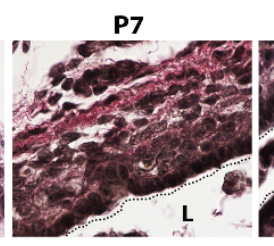

P10

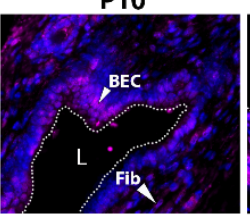

6 year

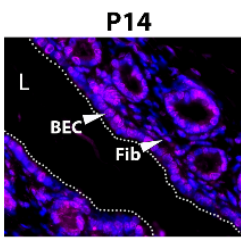

year old

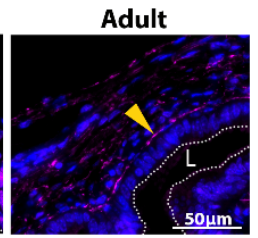

Adult

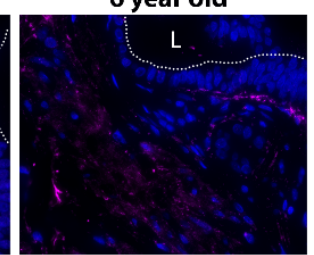

P14

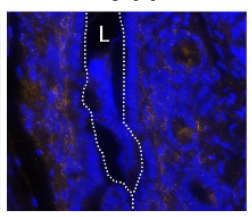

P14

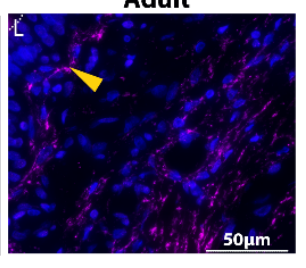

Adult

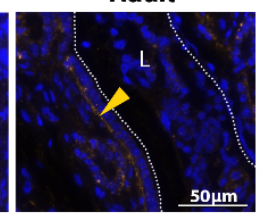

Adult

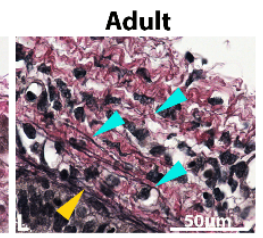

Figure 1: EFEMP1 expression in postnatal development of the mouse and human extrahepatic bile duct.

(a) Representative images of EFEMP1, elastin and elastic fiber staining in neonatal $(P 2,5,7,10,14)$ and adult mouse extrahepatic bile duct as well as human extrahepatic bile duct from individuals aged 3 days old, 6 months old, 3 years old, 6 years old and 19 years old (adult). White arrows show biliary epithelial cells (BEC) and fibroblasts (fib) expressing EFEMP1. Yellow arrows show EFEMP1 localization under the biliary epithelial cell monolayer. (b) Representative images of elastin staining in mouse samples. In all immunohistochemistry staining, nuclei are stained with DAPI (blue). Yellow arrow shows elastin localization under the biliary epithelial cell monolayer (c) Representative images of elastic fiber staining, with stained elastic fibers in black (teal arrows), nuclei in dark brown and collagen in pink. Yellow arrow shows elastic fiber localization under the biliary epithelial cell monolayer. In all images, dotted lines and $\mathbf{L}$ denote lumens. For mice, 3-4 samples were stained for each age. Size bars $=50 \mu \mathrm{m}$. 
a
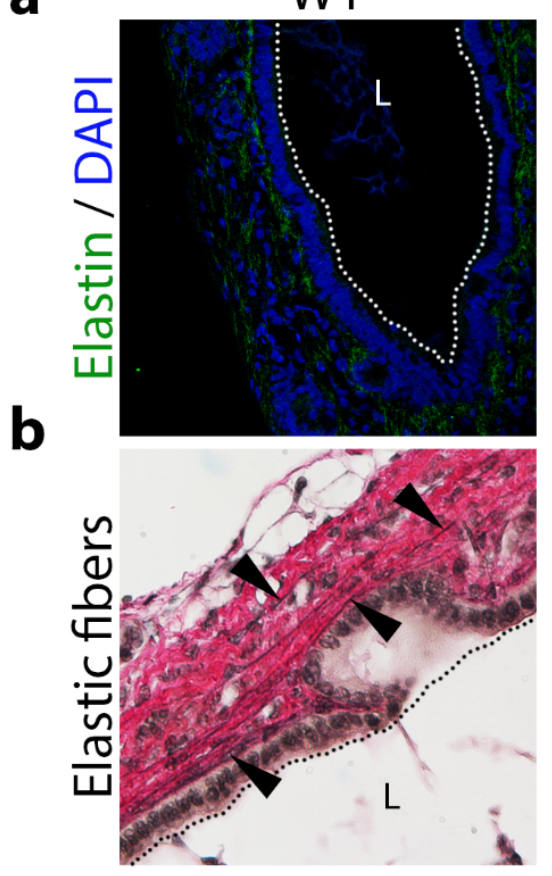

C

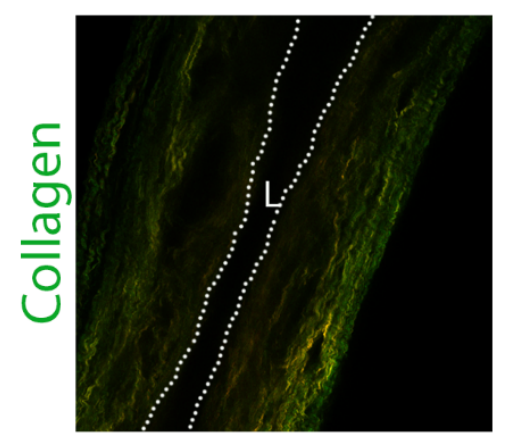

Efemp $1^{+/-}$
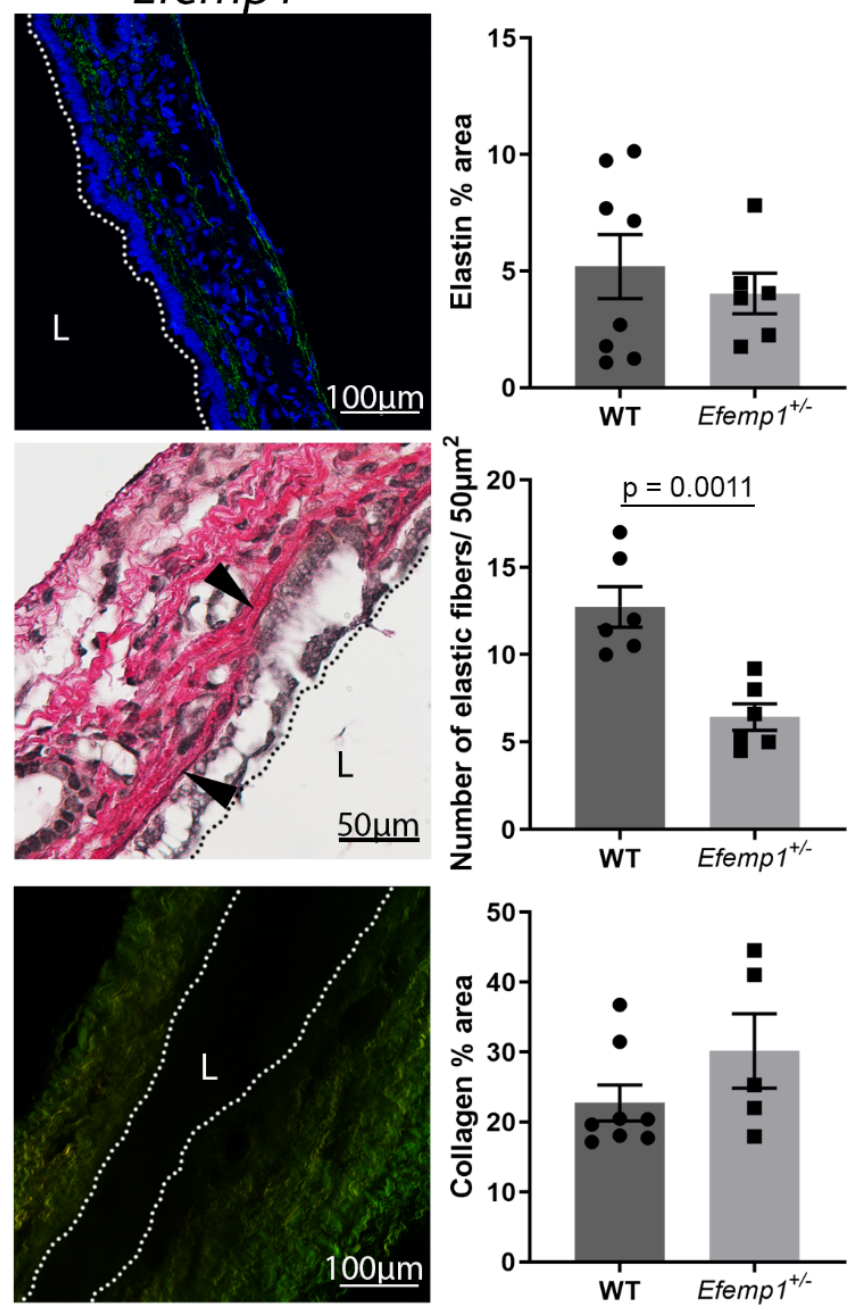

Figure 2: Elastic fibers but not elastin expression are reduced in adult $E f e m p 1^{+/}$extrahepatic bile ducts.

(a) Representative staining of elastin in WT and Efemp1 $^{+/}$extrahepatic bile ducts and quantification of percent area of staining. $\mathrm{P}=0.5264$ (b) Representative elastic fiber staining of $\mathrm{WT}$ and $E$ femp $1^{+/}$ extrahepatic bile ducts and quantification of the number of elastic fibers per $50 \mu \mathrm{m}^{2}$. (c) Representative images of collagen by SHG imaging in WT and Efemp1 ${ }^{+/-}$extrahepatic bile ducts, with quantification of percent area of signal. Forward signal in red and backward signal in green. $\mathbf{P}=0.1843$. Dotted lines and $\mathbf{L}$ denote lumens. 6-8 ducts were analyzed per genotype. Data shown are mean \pm SEM, significance determined by unpaired T-test. 
a

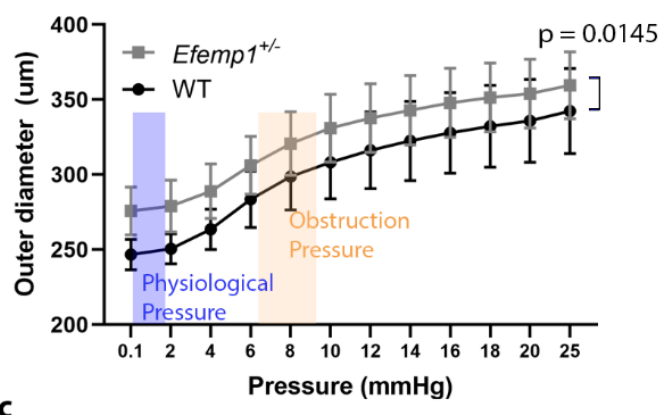

C

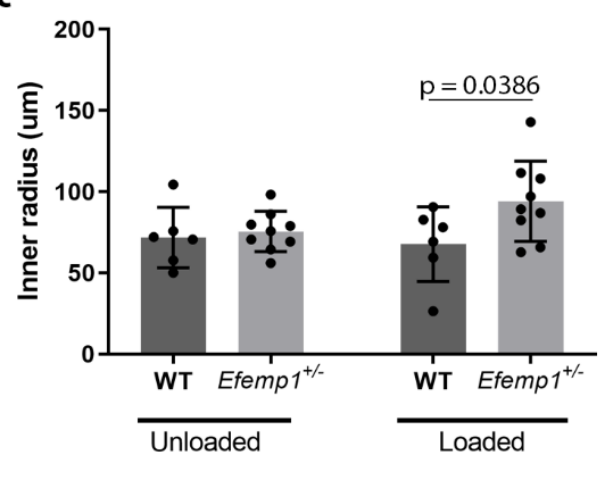

b

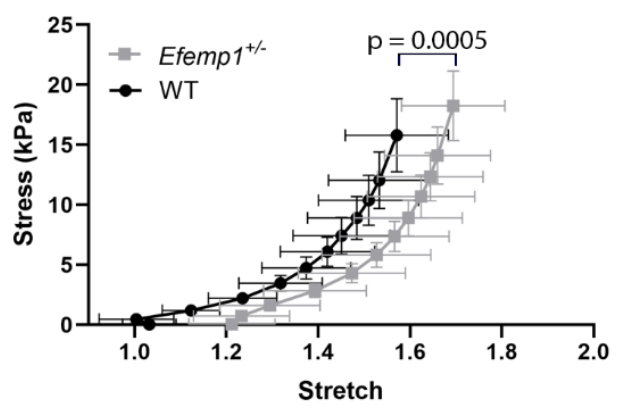

d

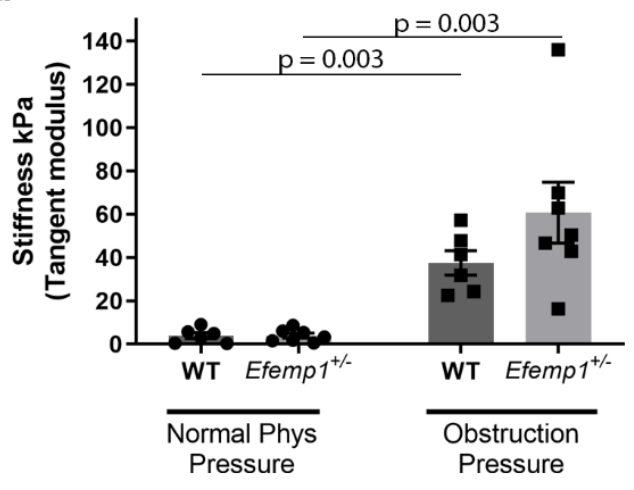

Figure 3: Mechanical properties measured by pressure myography are altered in Efemp $1^{+/-}$extrahepatic

bile ducts. (a) Measured outer diameter of extrahepatic bile ducts at increasing pressures. Physiological and obstruction pressures derived from the literature are indicated on the graph. (b) Calculated stress and stretch of WT and $E f e m p 1^{+/-}$extrahepatic bile ducts under increasing pressure in 2-mm increments from 0-20 mm Hg. Significance shown is for stretch. No difference in stress was observed, $p=0.057$. (c) Measured inner radius of unpressurized (unloaded) and pressurized (loaded; around $0.1 \mathrm{mmHg}$ ) WT and Efemp $^{+/-}$extrahepatic bile ducts. There is no difference between WT vs Efemp ${ }^{+/-}$in the unloaded state, $p=0.9265$ (d) Calculated tangential modulus/stiffness at normal physiological pressure $(2 \mathrm{mmHg}$ ) and obstruction pressure $(12 \mathrm{mmHg})$ in WT and $E f e m p 1^{+/-}$extrahepatic bile ducts. No significant difference is observed comparing WT vs Efemp1 $1^{+/-} \mathrm{p}=0.5144$ at both normal and obstruction pressures. 6-7 ducts were analyzed per genotype. Data shown are mean \pm SEM, two-way ANOVA with a Šidák post-hoc test statistical test. 


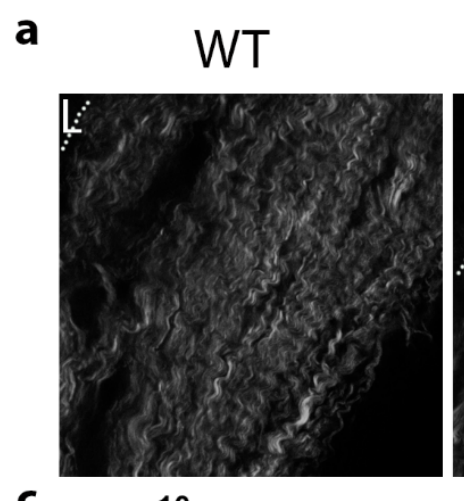

\section{Efemp $1^{+/} \quad$ b}
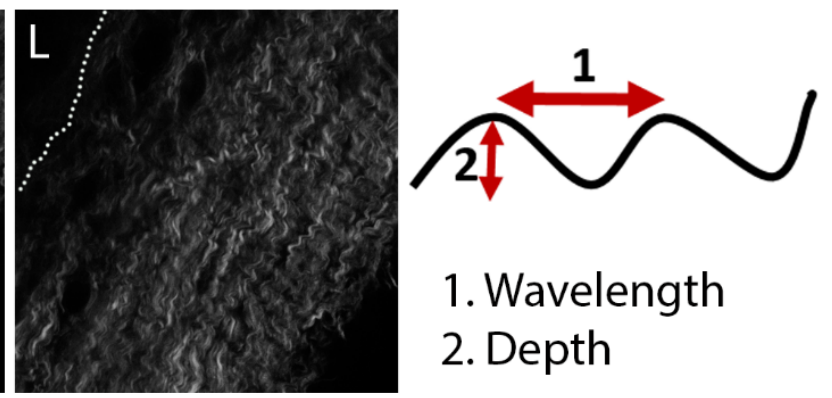

\section{Wavelength}

2. Depth

C
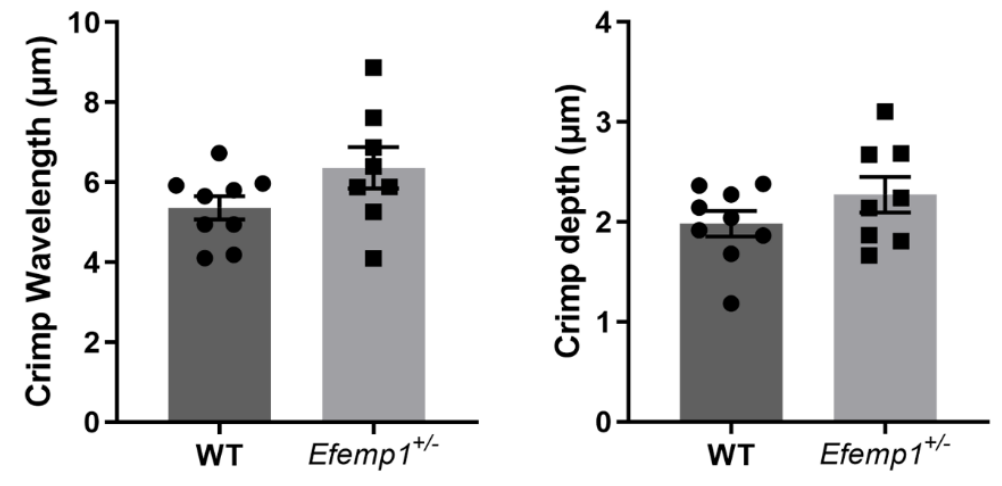

Supplemental Figure 1: No difference in collagen fiber crimping between WT and Efemp1 ${ }^{+/-}$extrahepatic

bile ducts. (a) Representative images of SHG microscopy used for fiber analysis. Dotted lines and $\mathbf{L}$ denote lumens. (b) Schematic of fiber crimp wavelength and depth measurements used for analysis. (c) Quantification of crimping. There is no difference between WT and Efemp1 $1^{+/-}$crimp wavelength $(p=$ 0.14041 ) or depth ( $p=0.1988$ ). For each analysis, 15 crimps per image were analyzed in 3 images per duct. 8-9 individual ducts were examined per genotype. Data shown are mean \pm SEM with each point representing the mean of a different duct. Significance was determined by unpaired T-test. 

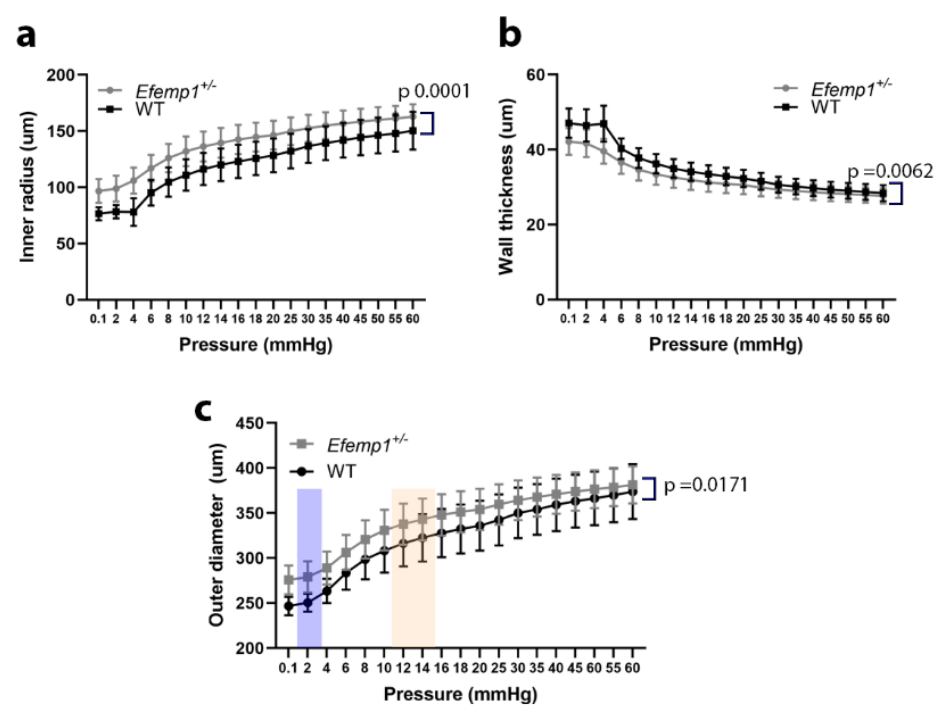

Supplemental Figure 2: Altered circumferential mechanics of ffemp1 $^{+/-}$extrahepatic bile ducts. (a) Measured inner radius of extrahepatic bile ducts at increasing pressures. (b) Measured wall thickness of extrahepatic bile ducts at increasing pressures. (c) Measured outer diameter of extrahepatic bile ducts at increasing pressures. Pressure was increased in 2-mm increments from 0-20 mm Hg followed by 5-mm increments (from 20-60 mmHg). 6-7 individual extrahepatic bile ducts were tested per genotype. Data shown are mean \pm SEM, two-way ANOVA with a Šidák post-hoc test statistical test. 